Revista Herencia Vol. 28 (2), 81-92, 2015

Recibido 31-08-2015 Aprobado 19-10-2015

\section{Patricia Sedó}

Masis. Docente Catedrática de la Escuela de Nutrición de la Universidad de Costa Rica. Cuenta con una Licenciatura en Nutrición UCR, Maestría Académica en Gerontología de la UCR y Maestría Profesional en Administración Educativa de la Universidad Latina.

Labora en la UCR desde 1989 dedicada al estudio de los alimentos y las tradiciones alimentarias de Costa Rica, así como en la atención nutricional de población adulta mayor. Desde el 2003 es responsable del proyecto de Trabajo Comunal Universitario en el tema de conservación y revitalización de la cocina tradicional costarricense. patricia.sedo@ucr.ac.cr

\section{UN ACERCAMIENTO A LA ESENCIA DE LA COCINA PATRIMONIAL de Costa Rica}

\begin{abstract}
Resumen
La cocina patrimonial de Costa Rica es una manifestación de interculturalidad. La herencia de saberes y prácticas asociadas con la producción, selección, preparación, conservación y consumo de alimentos, y el deseo de innovación a partir de ingredientes que tienen un connotado valor cultural y nutricional, muestran el proceso de construcción colectiva de una cocina nacional integrada por cocinas regionales, diferenciadas, dinámicas, diversas, ricas en sabores, aromas y texturas. Se evidencia en las últimas décadas un cambio negativo en los patrones alimentarios, dejando de lado la dieta tradicional, con un impacto en la salud de la población costarricense. Existe el interés por conservar las tradiciones alimentarias, pero son necesarios el conocimiento y las habilidades para elaborar las comidas y bebidas que forman parte del patrimonio alimentario. La desinformación principalmente por parte de las nuevas generaciones, un menor acceso a ciertos ingredientes, el sentimiento de nostalgia por una cocina ancestral que queda en la memoria colectiva y en recetarios, y los cambios en el estilo de vida vinculados con la globalización, el desarrollo alimentario industrial y comercial, las nuevas tecnologías y la sobrevalorización de lo foráneo hace que se enfrentan retos importantes en la conservación y revitalización de la cocina patrimonial en este país.
\end{abstract}

Palabras claves: cocina patrimonial, gastronomía, cocina costarricense.

\section{Abstract}

The traditional food practices of Costa Rica is a manifestation of interculturality. The heritage of knowledge and practices associated with production, selection, preparation, conservation and consumption of food, and the desire of innovation from ingredients that have a notorious cultural and nutritional value, show the process of collective construction of a national cuisine comprising regional cuisines, differentiated, dynamic, diverse, rich flavors, aromas and textures. It is evident in recent decades a negative change in eating patterns, leaving aside the traditional diet, with an impact on the health of the Costa Rican population. There is interest in preserving food traditions, but knowledge and skills are necessary to prepare food and drinks that are part of the food heritage. Misinformation mainly by new generations, less access to certain ingredients, the feeling of nostalgy for ancient cuisine that is in the collective memory and cookbooks, and changes in lifestyle linked to globalization, food industrial and commercial development, new technologies and the value of the foreign makes that major challenges in the preservation and revitalization of heritage cooking in this country face.

Keywords: traditional food, gastronomy, cooking Costa Rican. 


\section{Introducción}

La cocina forma parte del patrimonio cultural inmaterial de los pueblos y fortalece la identidad o sentido de pertenencia. La misma se caracteriza por una construcción colectiva de un fenómeno dinámico que tiene significación simbó lica para los individuos que la viven, la comparten, y buscan estrategias para su proyección y conservación (Dormaels, 2013; Torres et al, 2004).

La alimentación tradicional forma parte del patrimonio cultural y, a pesar de su intangibilidad, dispone de un corpus patrimonial tangible complementario asociado con la satisfacción de la necesidad de alimentación. Incorpora prácticas, representaciones, expresiones conocimientos y habilidades que a su vez $\mathrm{se}$ relacionan con espacios fisicos y un rico simbolismo cultural.

En la cocina, los espacios fisicos y familiares se entrelazan con alimentos, artéactos y utensilios que los indiv (Alvarez, 2002; UNESCO, 2003).

La cocina ubica a las personas en un espacio geográfico en el ámbito doméscúmulo de tareas que se realizan en la vida cotidiana y festiva.

Por otro lado, la intangibilidad del patrimonio alimentario se asocia más a la transitoriedad que a la inmaterialidad, puesto que en las tareas necesarias y simbólicas que las personas enfrentan un conjunto de actividades materiales mismo se que se enmarcan en un siste ina sociocultural bastante complejo. nización. De esta forma la alicantación va más allá de motivos estrictamente nización. De esta forma, la alimentación va más allá de moutivo

En la cocina las personas experimentan sensaciones diferentes, con una estimulación total de los sentidos, la interacción con otros, y la evocación de recuerdos de alto significado sociocultural.

Emociones y sensaciones se conjugan con una explosión de sabores y aromas que quedan impregnados en la memoria colectiva (Unigarro, 2010).

Ante la relativa escasa información actualizada sobre la cocina patrimonial disponible en el ámbito nacional se propuso realizar el presente estudio, cuyos objetivos han sido explorar las percepciones sociales sobre la cocina patrimonial costarricense e identificar las principales características de la misma.

Se procedió a realizar una consulta a 125 personas adultas residentes en el Gran Área Metropolitana miembros de comunidades, instituciones y org en el Gran relacionadas con la seguridad alimentaria y nutricional gastronomía, cultura turismo. Las personas consultadas tenían un vínculo con el proyecto de TCU-486 sobre cocina tradicional costarricense y el proyecto EC-317 sobre el turno com espacio de manifestación cultural gastronómica, ambos proyectos coordino por la Escuela de Nutrición de la Universidad de Costa Rica. La consulta se realizó durante el período 2011-2014.

El estudio es de enfoque cualitativo, para lo cual se aplicaron entrevistas grupales e individuales con el uso de una guía semiestructurada.

\section{La comida, la cocina y la gastronomía}

Para profundizar en la conceptualización de la cocina patrimonial emergen diferencias en las personas consultadas en lo que se entiende por "comida", "cocina" y "gastronomía".

La cocina, como actividad de alta significancia sociocultural, encierra un conjunto de saberes aquilatados a lo largo de la historia de la humanidad, así como un amplio conocimiento ecológico que muestra alto dinamismo, pero que conserva determinadas prácticas identificadas como seguras y necesarias de mantenerse, por lo que cobra valor la herencia a las nuevas generaciones (Torres et al, 2004)

Al hablar sobre la cocina patrimonial de Costa Rica, los términos "cocina" y "comida" sobresalen en el discurso de las personas consultadas. Lo anterior es coincidente con un reciente estudio Más que fuentes de sustancis nu

Más que fuentes de sustancias nutritivas, los alimentos englobados como un conjunto de ingredientes y consumen en el con(a) sersonas se sienten identificadas y amiliarizadas.

Para Álvarez (2002:11) "comer implica un hecho social complejo que pone en escena un conjunto de movimientos de producción y consumo, tanto material como simbólico, que son diferenciados y diferenciadores".

"Nuestra comida", "la comida tica" o "lo que comemos siempre" evidencia la estrecha relación de estos términos con la tradición y la necesidad de su apropiación. Por su parte, la "comida popular" se relaciona con el consumo cotidiano de alimentos, los cuales forman parte del patrón alimentario de una mayoría que, en el caso de Costa Rica, podría ser el gallo pinto según las personas entrevistadas. Ramírez (2002) indica que lo considorado en las cocinas populares insinúa la posición ocupada en la jerarquía social y la trayectoria en la historia nacional. La cocina como manifestación sociocultural se considena como un sistema muy complejo que a arca múltiples dimensiones de la vida del ser humano. Es así como lo popular lo criollo lo campesino, lo típico y lo tradicional emergen en

La comida o cocina tradicional se asocia con aquellos alimentos y prácticas alimentarias que tienen un simbolismo diferenciado, segun lo refieren las personas entrevistadas, y que los acerca a sus raíces históricas familiares.

El conocimiento y las habilidades culinarias de la cocina tradicional se añoran, se disfrutan y se tratan de dar a conocer a otros, con lo cual la herencia de generación en generación es la clave para que se mantenga en la memoria colectiva. Del mismo modo que no hay una sola tradición, también se puede afirmar que no hay una sola cocina en el país (Unigarro, 2010).

Lo popular tiene estrecha relación con la cocina tradicional o la cocina patrimonial. Como sustento de lo tradicional, muchas de las preparaciones que forman parte de la cocina popular se fundamentan en las recetas que se han heredado de generación en generación y no tanto de la gastronomía promovida en los sectores de mayor poderío económico, donde se busca más lo foráneo o su dieta no necesariamente está fundamentada en los alimentos locales (Ramírez, 2002). En el caso de Costa Rica no se puede hablar de una sola cocina. Variables tales como la producción y disponibilidad de alimentos por regiones, el impacto de las migraciones internas, el aporte de otras culturas y las transformaciones socio históricas han contribuido y continúan enriqueciendo la conformación de variadas formas de expresión culinaria, que a su vez constituyen una cocina nacional con identidad propia.
Foto 1 Feria del Café Frailes 2014. Gimen Cortés. 
Las cocinas regionales nos sitúan en un espacio geográfico determinado y, en el caso de Costa Rica, las diferencias en las tradiciones alimentarias no necesariamente coinciden con la división político administrativa. En este caso corresponde a regione culturales claramente establecidas, como la delimitación del norte y sur del país, el Valle Central, la zona caribeña, las zonas costeras y los territorios indígenas, por ejemplo. En el caso del término "gastronomía", en las personas consultadas se evidencia su relación con lo gourmet, lo foráneo o aquello focalizado en un restaurante hotel, lo cual no necesariamente tiene rasgos identitarios.

Al respecto se señala la marginación de las construcciones colectivas culinarias y la participación de un solo cocinero o de un grupo selecto quienes, haciendo alarde de sus conocimientos en gastronomia, supuestamente innovan o crean nuevos platos con ingredientes locales o nuevos conceptos que no tienen idenpuesta de un souflé de camote ouna terrina de pejibaye. puesta de un souflé de camote o una terina de pejibaye.

" un espacio "artificial, se prom ena con poca información, una inagen distorsionada de la cocina tradicional, con la midas que no forman parte del acervo cultural del país pero que se denomina

A pesar de que el término "gastronomía" es ampliamente utilizado en la comunidad internacional, y se reconoce la relevancia de una visión más amplia integral, la famillaridad con el termino y su identidad con las tradiciones alimentarias no es muy cercana para Costa Rica, según las personas entrevistadas. no a Tronomí Costarricense Sostenible y Saludable (2011) promovido

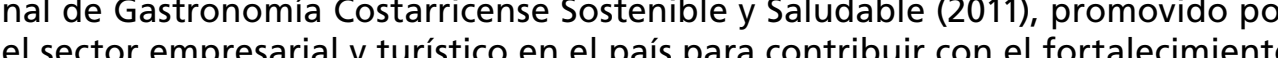
de la empresarialy turistico en el pais para contribuir con el fortalecimiento cómo formar a las personas encargadas de la cocina y del servicio al cliente sobre puesto que la oferta no las caracteristicas esenciales de la cocina patrimonial, pues

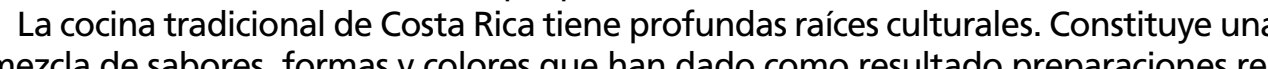
mezcla de saboriones reconocides - En comparación con otros países de la región, Costa Rica dispone de una cocina relativamente sencilla en términos de ingredientes, condimentación y técnicas culinarias. A pesar de ser un país tan pequeño en extensión geográfica, presenta variaciones gastronómicas en sus regiones, determinadas principalmente regiones, determinadas principalment
por la rica biodiversidad alimentaria. por la rica biodiversidad alimentaria. Varios son los platillos y bebidas que
se mencionan como parte del menú se mencionan como parte del menu la tortilla, los tamales, los picadillos, la olla de carne, diversas preparaciones a base de maíz, las bebidas a base de frutas o cereales, los guisos, los dulces o conservas con miel de caña (tapa de dulce), panadería y repostería criolla.
Cocinar, comer y alimentarse. Visiones de la actividad de alimentación y la dieta tradicional en Costa Rica

La dieta tiene una relación estrecha con la evolución humana y los cambios en el estilo de vida de los individuos y las poblaciones. En un mundo globalizado, donde las fronteras se desdibujan y se desterritorializan los espacios, lenimato, con nuevas formas de inertir su anonimato, con nuevas foŕltiples necesidades, entre para atender las mútiples necesidades, Para muchos, la intoraccior

Para muchos, la inceración con otros se loformas la aplicación dé nuevas tecnologías y de aferrarse a sus ráces y a sus manifestaciones culturales, como elementos identitarios diferenciadores en una sociedad de agresivo consumo (Traversa, 2002).

La alimentación, a la que nuestros ancestros de-

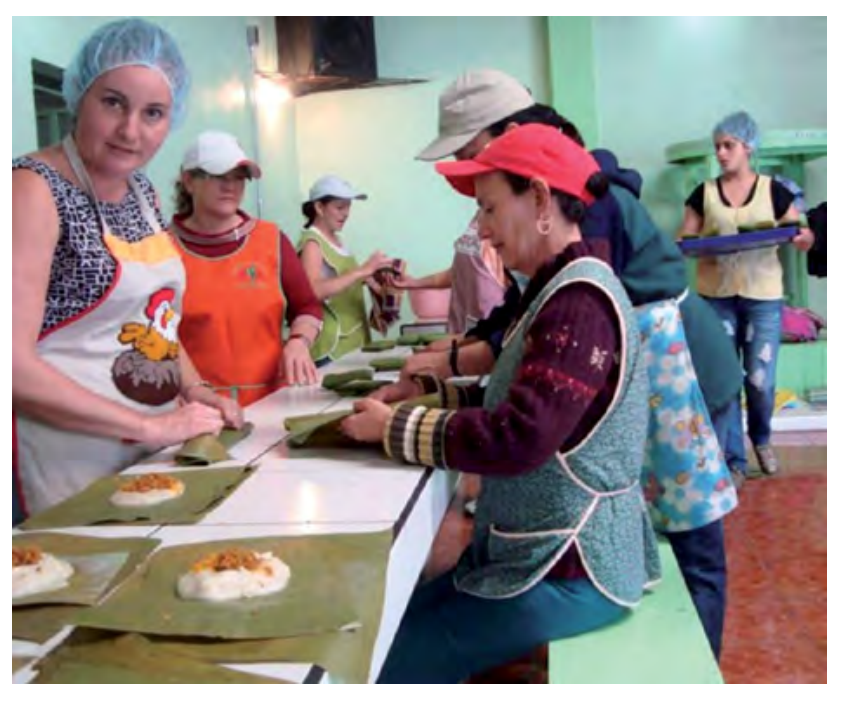

dicaban gran parte del tiempo, dado que era necesario buscar los productos alimentarios, producirlos, cocinarlos, enseñar a otros a procesarlos y se disfrutaba el consumo (Torres et al, 2004).

Se presenta el agravante que otras actividades compiten con el tiempo dedicado a la alimentación, con lo cual la tendencia actual es buscar la simplificación de las tareas mediante la adquisición de alimentos ultra procesados o listos para el consumo, lo cual resulta en un ciclo donde hay una respuesta de la industria y del medio para solucionar la vida moderna pero, a su vez, las personas reaccionan ante la publicidad que les hace creer que ese es el camino (Organización Panamericana de la Salud, 2015). La mayor dependencia de las personas por adquirir alimentos listos para conuno contribuye a la perdida de la ta y cióntos saborizantes artificiales que se dicionales (Organización Panamericana de la Salud, 2015).

La marginación de las dietas tradicionales representa un alto riesgo no solo para la salud, sino para la cultura alimentaria y la seguridad alimentaria y nutricional de las poblaciones. Existe el riesgo de perder el conocimiento a yumulado cional de las poblaciones. Existe el riesgo de perder el conocimiento acumulado disponib de la historia sobre el aprovechamiento de los recursos alimentarios utilizar lo disponible (Mateos y Rodríguez, 2010).

La tendencia a la homogenización de la dieta y la pérdida de la práctica de aprovechar los recursos alimentarios naturales y procesarlos en casa para su consumo afecta negativamente la nutrición de las personas.

Aunque se ha evidenciado que la alimentación es una de las actividades que muestra cambios más lentos en la vida humana, se han determinado aceleradas modificaciones en los patrones de alimentación en los últimos cien años, las cuales tienen una relación directa con el estado de salud y bienestar de las poblaciones en todo el mundo, y Costa Rica no es la excepción.

La especie Homo sapiens tuvo una adaptación alimentaria impresionante, con la capacidad de tener una dieta tan variada como lo es la diversidad cultural que puebla
Foto 3. Elaboración de tamales. 
el planeta. Logró transformar los alimentos para una mejor digestibilidad, y con e descubrimiento del fuego y la perfección del arte de cocinar fue incorporando nuevo recursos alimentarios a su alimentación diaria, lo cual contribuyó no solamente a su sobrevivencia, sino también a la posibilidad de crear nuevas formas de alimentación como expresión cultural y diferenciación entre grupos (Mateos Rodríguez, 2010). Dietas tradicionales en muchos pueblos han sufrido cambios negativos importantes, con una menor disponibilidad de alimentos nutritivos y un mayor consumo de productos ultraprocesados de escaso valor nutricional. Un ejemplo de lo anterio son las transiciones en la dieta tradicional mexicana y su impacto en la salud y calidad de vida, donde el alto consumo de bebidas azu caradas tiene una relación directa con la epidemia de obesidad que agobia a la población (Serrano y Cussó, 2012). La ubicación planetaria en el trópico, hace que en Costa Rica se disponga de una alta biovidersidad alimentaria, con abundancia de productos vegetales, sin embargo el consumo de frutas y vegetales es limitado, y actualmente algunas partes de plantas conestibles son consideras es el caso de las senillas, flores y brotes tien o (cono y cos co, 2014). que forman pas semillas, flore y breses tiennos (conocidos como queli

volućn en la alime teniendo los recursos suficientes para mantener una die come para sobrevivir aun teniendo los recursos suficientes para mantener una dieta variada y satisfactoria.
Además, se ha perdido la relevancia la convivencia y comensalidad, tan arraigada Sobre la alimentación,

ción, las personas consultadas establecen una diferenciación y alimentarse; además se presenta un concepto relativamente abstracto de la alimentación como un derecho humano fundamental.

Foto 4. "Crear nuevas foras de alientación cono expresión cultura

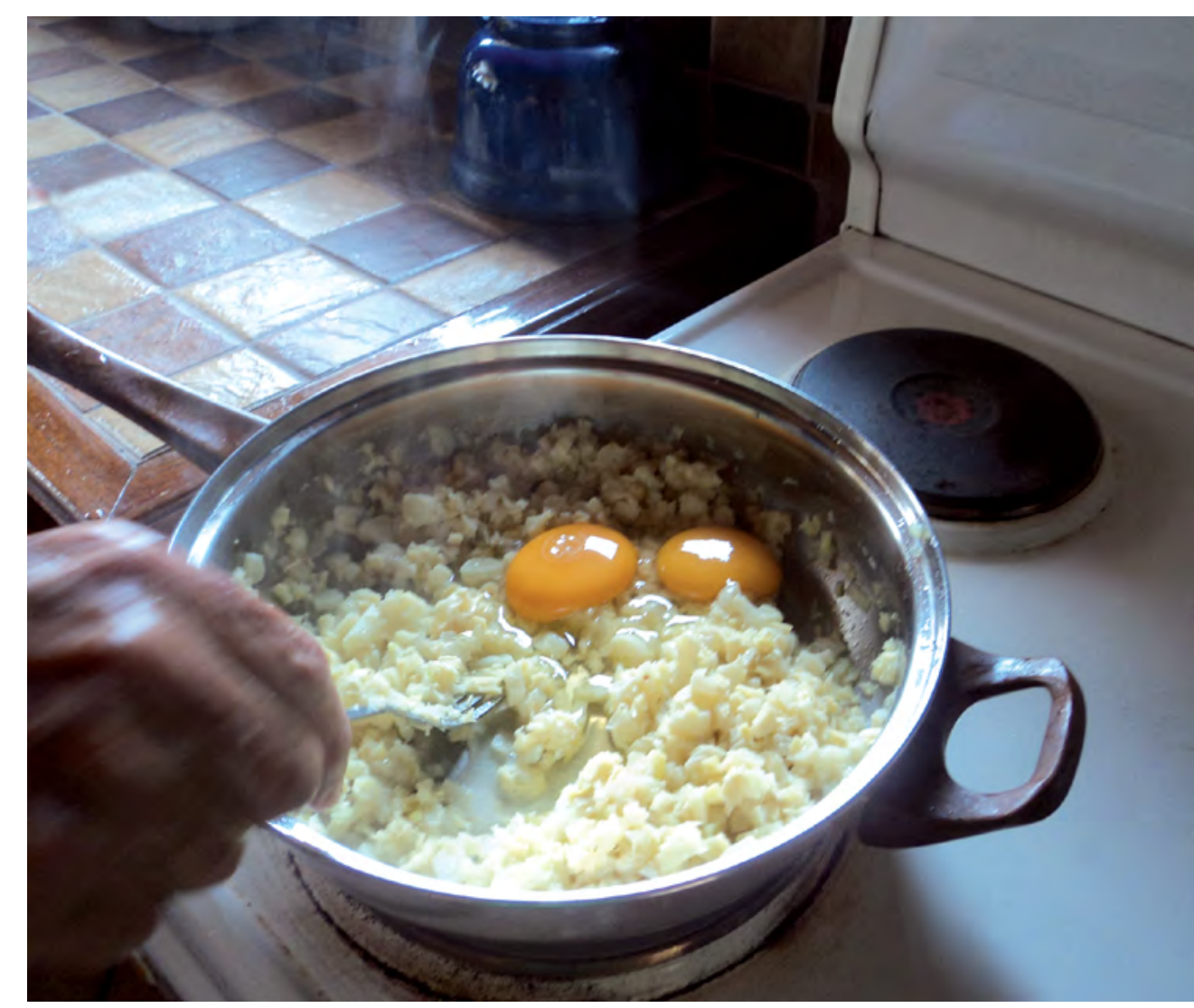

Se percibe que "comer" es el acto cotidiano de consumir los alimentos que las familias tienen a su alcance; mientras que "alimentarse" se refiere al seguimiento de una dieta saludable y balanceada, para lo cual las familias requieren de educación y mayor poder adquisitivo.

Por otro lado, una alimentación saludable se asocia con salud y belleza corporal, idea fomentada por los medios de comunicación masiva, con lo cual se convierte para muchos en una utopía o un asunto de estética corporal.

En la visión de "comer" como un acto de sobrevivencia y la consecución de los alimentos básicos, sobresale en el discurso los alimentos de consumo popular que forman parte de las tradiciones alimentarias, entre ellos los frijoles o la mezcla de tales como la producción y el acceso. Al respecto menciona una persona:

"No somos capaces de producir ni el gallo pinto en las cantidades que la población requiere, y dependemos de la compra a otros países (...) Los agricultores están falto mu golpeados por las malas pollticas del gobieno, y por pobreza o por

En relación con la visión de "alimentarse" se determinan discursos encontrados respecto a cómo lograr una buena alimentación y el uso de alimentos tradicionales. Para una gran mayoría, alimentarse con gallo pinto es una forma barata nutritiva una resolver la necesidad de alimentarse y nutrirse. Sobre este tema comúc una señora: "El alimise bien no es la comida chatarra o estar comiendo fun a de horas, no de una forma do es la col nutirse. Sobre este temarefleja en su rostro cuando tiene una buena alimentación".

Al hablar sobre estos temas, emergen preocupaciones respecto a los cambios que se perciben en el acceso y las formas de alimentarse las personas en el mundo actual. El acceso a los alimentos de consumo básico se ve limitado por fluctuaciones en los precios y bajos salarios minos, con lo cuar existe la incapacidad de las personas de bajos recursos el poder ad conir los alir

El alto costo de ciertos ingredientes para elaborar un platillo tradicional provoca que las familias se vean limitadas a prepararlo, con lo cual se pierde la práctica en el seno familiar.

Al respecto indica un señor:

"Los alimentos son cada vez más caros, y alimentarse de forma sana es poco factible para las familias costarricenses", "hacer tamales o la tradicional olla de carne sale sumamente caro, por lo que ya no es posible hacer comidas nuestras".

Sobresale también la preocupación por la incapacidad de las familias para auto abastecerse de los alimentos que requiere para su alimentación diaria, principamente en el entorno urbano, y la dependencia a comprar la comida.

Entrevistados de zonas rurales y dedicados a la producción agrícola también refieren que, a pesar dedicarse a la siembra de alimentos y sentirse en mejores condiciones en comparación con las personas que viven en la ciudad, enfrenta grandes crisis, por cuanto la siembra de productos sufre el impacto del cambio climático y su trabajo no es recompensado económicamente, con lo cual existe un desestimulo y enfrentamiento a un círculo de pobreza. 
La imposibilidad de producir lo que se requiere para comer, hace pensar que deba "volverse la mirada a lo que se comía antes", haciendo alusión de la relevancia de sacar un mayor provecho de los productos disponibles, acrisolar el conocimiento para la recolección de comida en el monte, y desempolvar las recetas de antaño caracterizadas por un amplio uso de alimentos considerados como "de pobres", como es el caso del guineo, la verdolaga, el culantro coyote,
las flores de ayote o los pitos o flores de poró.

Sobre el tema de producción de alimentos, un agricultor consultado mencionó lo siguiente:

"el cambio climático y la contaminación afectan la producción de alimentos y las fuentes de agua. Conforme pasa el tiempo los problemas probablemente serán mayores (...) la agricultura es una actividad riesgosa, dado que los créditos no son tan accesibles, los precios pagados por los productos por intermediarios son muy bajos y no compensan todo lo invertido en el campo, y con los cambios en el clima existe un alto riesgo de perdida, sea por sequia o excesivas lluvias que que mis hijos estudien para que se dediquen a otra cosa".

La calificación de "alimentos de pobres", "alimentos del monte", "alimentos de nuestras abuelas", "alimentos de siempre" "comida de polos" o "cocina de turno pueblerino" nos hace reflexionar acerca de idea despectiva que tiene de ciertos alimentos que ocupan un lugar especial en la cocina tradicional.

Sobre la diferenciación entre la comida de consumo cotidiano y en el contexto festivo, se citan productos específicos, como los tamales para la época navideñ - la cocina de turno, el picadillo de chayote y zanahoria para consumo frecun 作 y el picadillo de arracache para una fiesta, o la chicha de maíz para celebrar un
acontecimiento especial.

Por otro lado, sobresale otra preocupación referente a micronegocios o empresas familiares, los cuales son considerados espacios donde es posible mantelorales, sodas de pueblo, pulperías, trapiches y fábricas arter son las panaderias

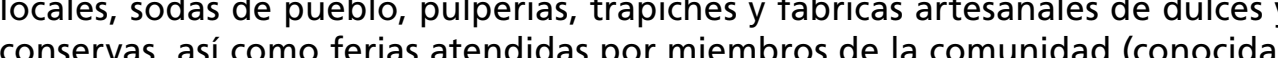
popularmente como turnos).

La competencia con cadenas comerciales grandes, los requisitos sanitarios percibidos como restrictivos y veces imposibles de cumplir, los altos precios de insumps, y poco acceso a nueva tecnología impactan de manera importante estas actividades. Se percibe que las políticas y acciones institucionales no tienen mucha claridad respecto a la salvaguarda de estos espacios, y en un contexto de mercado de agresiva competencia se presentan inequidades. Sobre el tema anterior se comentaba: "mantener una pulpería o un negocio pequeño es muy dificil en este pais, comentaba: "mantener una pulperia o un negocio ayuda a los grandes, no a los pequeños".

Otra preocupación que sobresale en el discurso son los cambios en los patrones de alimentación y en las tradiciones alimentarias donde, a pesar de que se reconoce que existe un dinamismo en los grupos humanos que lógicamente impacta todas las dimensiones de la vida, en el caso de la alimentación los cambios se perciben como rápidos y negativos.

Sobre este tema se menciona: "el consumismo y la pérdida de las tradiciones alimentarias afecta la calidad de la alimentación en la población costarricense". Según las personas entrevistadas, existe un alto riesgo de considerar de forma nostálgica las tradiciones alimentarias como actividades del pasado, sin esperanza de recuperación de los saberes o desinterés de mantener vivas las tradiciones, sea porque no se tiene el conocimiento para su perpetuación, o porque los nuevos estilos de vida imponen nuevas necesidades o dependencias.

En relación con lo anterior, una persona mencionó lo siguiente: "estamos enfrentando cambios drásticos en el estilo de alimentación, con menos interés por cocinar, y una dependencia a alimentos preparados y procesados industrialmente, que no forman parte de nuestra cultura."

En el estudio de las tradiciones alimentarias es necesario tener un panorama amplio de las actividades que se vinculan con la alimentación, incluyendo las tradiciones agroalimentarias, las prácticas de cocina y la comensalidad.

La alimentación, como manifestación cultural, entreteje múltiples tareas e involucra la participación comunitaria y la herencia ancestral. Está permeada por otras actividades humanas, y a traves de la comida es posible la expresión sociocultural. Al analizar el tema de la comida y las tradiciones alimentarias en Costa Rica, existe una percepción generalizada entre las personas consultadas sobre las transiciones relación con la práctica de cocinar, y la dedicación de menor tiempo a actividades relacionadas con la alimentación. relacionadas con la alimentación.

\section{La cocina patrimonial de Costa Rica, \\ más que recetas}

La herencia de las recetas de platillos tradicionales no debe quedarse en recetarios polvorientos. Es necesario motivar, principalmente a las nuevas generaciones, para que atesoren los conocimientos y habilidades necesarias para conservar y revitalizar la cocina tradicional.

Según las personas entrevistadas, la cocina patrimonial está íntimamente ligada a herencia, alta laboriosidad, uso de los recursos alimentarios disponibles en la localidad disfrute familiar, lo cual es coincidente con la investigación de Aguilar et al (2014).

La cocción a la leña y a fuego lento otorga un valor diferenciado a las comidas, con lo cual hace suponer que las técnicas de elaboración imprimen un sello particular, que lo diferencia de un producto industrializado. No obstante, si las ideas prede ingredientes que son difíciles de conseguir o de ingredientes que son dificles de conseguir o son caros, y que su elaboración es compleja, es necesario educar y crear nuevas de lo considerado como tradicional.

Se reconoce también que para elaborar comidas tradicionales se requiere conocimiento y práctica, y que las habilidades son acrisoladas por las mujeres dedicadas en su acrisoladas por las mujeres dedicadas en su vivas las tradiciones alimentarias.

Es así como otra estrategia necesaria es hacer un abordaje con enfoque de género $y$ aprender a disfrutar los sabores de la cocina patrimonial. Con el pasar del tiempo muchas de las
comidas y bebidas que han sido seleccionadas como representantes del menú típico de la tierra tica, pueden encontrarse en otros países

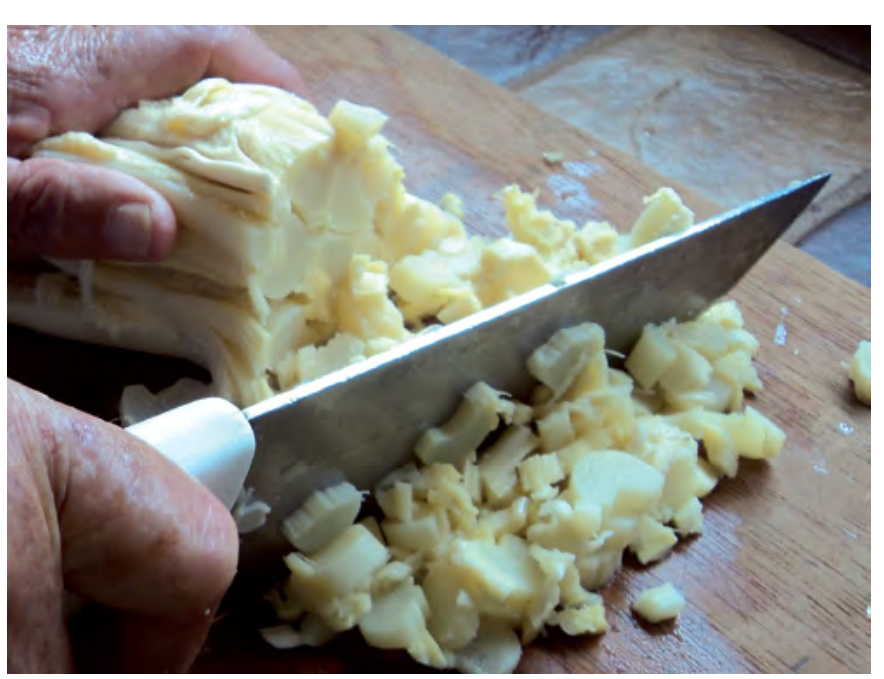




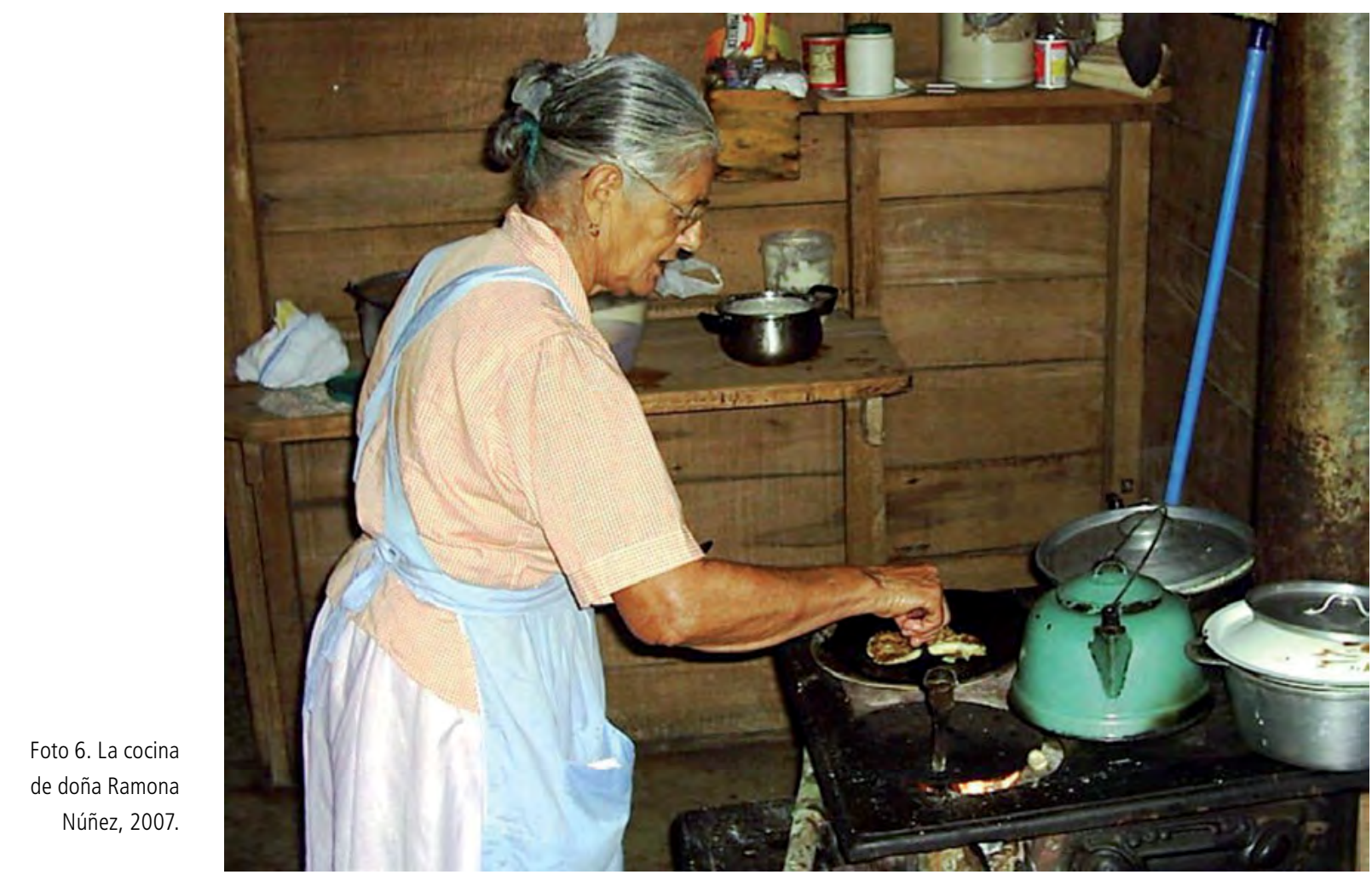

de la región con diferentes nombres o ciertos ingredientes. Ello nos recuerda que no estamos aislados, sino que hay una pertenencia a una región geográfica que comparte rasgos culturales.

Existen algunos elementos diferenciadores, entre los que destacan la tendencia a picar finamente los alimentos en la elaboración de guisos poco acuosos, lo que contribuyó a una forma muy particular de preparar los vegetales en Costa Rica que se denominó como "picadillos".

Otra característica propia de Costa Rica es la homogeneidad en la condimentación natural. Para resaltar el sabor de los platillos se utiliza predominantemente la cebolla, el chile dulce, el ajo y el culantro de castilla o de coyote que, en su conjunto, se conoce como "olores".

Los "olores" se utilizan prácticamente en todos los platillos. Estos productos se pican finamente y se sofríen en manteca 0 aceite antes de agregar el resto de los ingredientes.

Anteriormente la grasa para la cocción por excelencia era el sebo de res o la manteca de cerdo; posteriormente, con la explotación de la palma aceitera, se utilizó la manteca vegetal, la cual fue sustituida, en la década de los noventa, por el aceite vegetal como resultado de las campañas educativas para modificar los hábitos de alimentación como medida para la prevención de enfermedades cardiovasculares por parte del sector salud.

En cuanto a los platillos dulces, se usa la esencia de vainilla, el clavo de olor y la canela; aunque en tierras caribeñas es común el uso del jengibre, la nuez moscada, la jamaica y el coco.

Las sopas, arroces y platillos con carnes son variados. Entre los predilectos para las fiestas se destacan la sopa de mondongo y la de albóndigas, el arroz con pollo, la carne de res en salsa de tomate y el lomo relleno.
Lamentablemente, con el pasar del tiempo, ciertos platillos importados como lasagñas, hamburguesas y pizzas han desplazado estas preparaciones. Lo mismo sucede con los dulces y conservas tradicionales elaboradas con tapa de dulce, con un amplio aprovechamiento de los sobrantes de frutas disponibles en los solares, sustituidos por postres importados como Lisur o el cheese cake (Sedo, 2014).

Las preparaciones se han dejado de elaborar en las casas, aunque es común encontrarlas en restaugares que se resisten a perder la herenciaculinara.

\section{Comentarios finales}

La cocina patrimonial de Costa Rica es una manifestación de interculturalidad. La herencia de prácticas de producción, selección, preparación, conservación y consumo de alimentos, y el deseo de innovación a partir de ingredientes que tienen

un connotado valor cultural y nutricional, muestran el proceso de construcción colectiva de una cocina nacional integrada por cocinas regionales, diferenciadas, dinámicas, diversas, ricas en sabores, aromas y texturas.

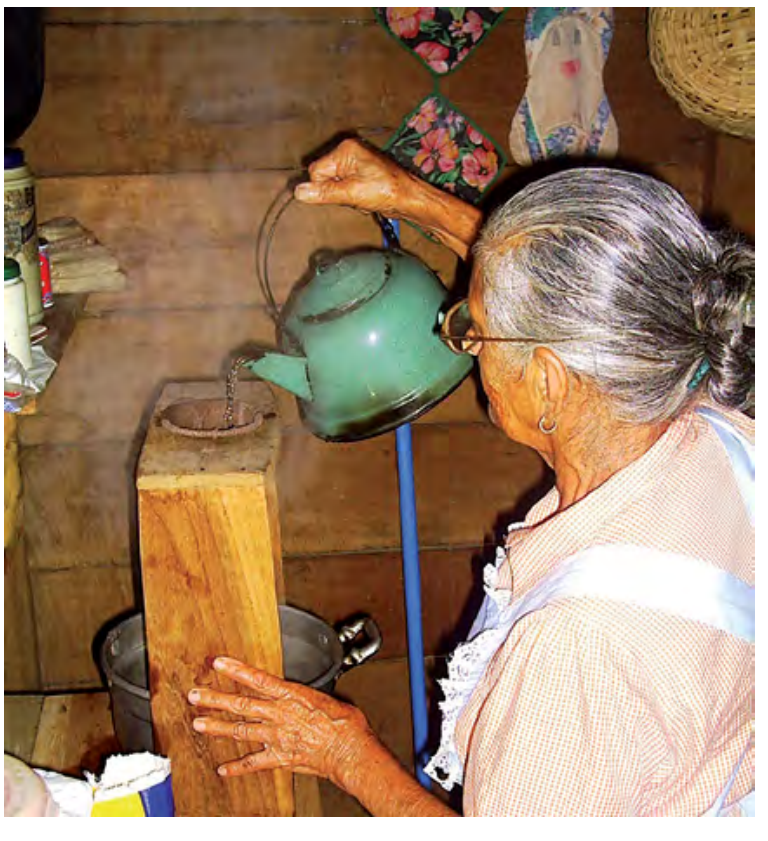

Los cambios en el estilo de vida y en los patrones de alimentación de la población ( conservación y revitalización de la cocina patrimonial.

Es necesario el conocimiento y la práctica para elaborar las comidas y bebidas que Es necesario el conocimiento y la practica para elaborar las comidas y bebidas que encuentro intergeneracional para continuar con el legado.

\section{Bibliografía}

Aguilar, Pamela; Arias, María José; Rojas, Cristina; Solano, Melissa (2015) "Aportes conceptuales y metodológicos para el fomento de la cocina tradicional costarricense como insumo en el desarrollo de políticas públicas e iniciativas nacionales, bajo un enfoque integral de la seguridad alimentaria y nutricional" San José: Seminario de Graduación para optar por la Licenciatura en Nutrición, Universidad de Costa Rica.

Álvarez, Marcelo (2002). "La cocina como patrimonio cultural (in) tangible". En: La cocina como patrimonio (in) tangible. Argentina: Comisión para la preservación monio Cultural No. 6: pp. 11-25.

Mateos, Ana; Rodríguez, Jesús (2010). La dieta que nos hizo humanos. España: Museo de la Evolución Humana.

Organización Panamericana de la Salud (2015) "Los alimentos ultraprocesados son motor de la epidemia de obesidad en América Latina". Dirección: http://www.paho. org/uru/index. php?option=com content\&view=article\&id=977: Ios-alimentos-ultraprocesados-son-motor-de-la-epidemia-de-obesidad-en-america-latina-senala-unnuevo-reporte-de-la-opsoms\&ltemid=340. Consulta: 11 de diciembre del 2015 .
Foto 7. Doña Ramoiñ̃ez, chorreando 
Ramírez Carolina (2002). "Comida regional como comida de pobres. Prácticas y representaciones culinarias en sectores populares de la ciudad de Posadas". En: La cocina como patrimonio (in) tangible. Argentina: Comisión para la preservación del Patrimonio Histórico Cultural de la Ciudad de Buenos Aires. Temas de Patrimonio Cultural No. 6: pp. 125-138.

Sedó, Patricia (2014). Pique olores, tacacos a la olla y a cocinar... Reflexiones sobre la cocina del costarricense. San José: Escuela de Nutrición, Universidad de Costa Rica.

Sedó, Patricia; Solano, Mayela (2014). Sembrar y comer alimentos con historia, sabor y nutrición. Reflexiones y propuestas para fortalecer la seguridad alimentaria y nutricional local. San José: Escuela de Nutrición UCR/Museo de Cultural Popular UNA.

Serrano, Eloi; Cussó, Xavier (2012). Soberanía alimentaria y cambios en la dieta en México 1961-2009. Una primera aproximación a partir de datos sobre consumo aparente de la FAO. España: Universidad Autónoma de Barcelona.

Torres, Graciela; de Zito, Madrid; Santoni, Elsa.(2004). “El alimento, la cocina étnica, la gastronomía nacional. Elemento patrimonial y un referente de la identidad cultural". Rev. Scripta Ethnologica (Núm. 26): pp. 55-66.

Traversa, Olivier (2002). "Modalidades discursivas de lo alimentario". En: La cocina como patrimonio (in) tangible. Argentina: Comisión para la preservación del Patrimonio Histórico Cultural de la Ciudad de Buenos Aires. Temas de Patrimonio Cultural No. 6: pp. 47-51.

UNESCO (2003). Convención para la salvaguarda del patrimonio cultural inmaterial. Dirección: http://unesdoc.unesco.org/images/0013/001325/132540s.pdf. Consulta: 11 de diciembre del 2015.

Unigarro Solarte, Catalina (2010). Patrimonio cultural alimentario. Ecuador: Fondo Editorial de la Cultura. Colección Cartografía de la Memoria, Número 4. 